\title{
Induction of otic structures by canonical Wnt signalling in medaka
}

\author{
Baubak Bajoghli • Narges Aghaallaei • Gerlinde Jung • \\ Thomas Czerny
}

Received: 7 April 2009/Accepted: 30 August 2009 /Published online: 16 September 2009

(C) The Author(s) 2009. This article is published with open access at Springerlink.com

\begin{abstract}
The Wnt family of signalling proteins is known to participate in multiple developmental decisions during embryogenesis. We misexpressed Wnt1 in medaka embryos and observed anterior truncations, similar to those described for ectopic activation of canonical Wnt signalling in other species. Interestingly, when we induced a heat-shock $W n t 1$ transgenic line exactly at $30 \%$ epiboly, we observed multiple ectopic otic vesicles in the truncated embryos. The vesicles then fused, forming a single large ear structure. These "cyclopic ears" filled the complete anterior region of the embryos. The ectopic induction of otic development can be explained by the juxtaposition of hindbrain tissue with anterior ectoderm. Fibroblast growth factor (Fgf) ligands are thought to mediate
\end{abstract}

Communicated by T. Hollemann

Baubak Bajoghli and Narges Aghaallaei contributed equally to this work.

Electronic supplementary material The online version of this article (doi:10.1007/s00427-009-0302-z) contains supplementary material, which is available to authorized users.

\footnotetext{
B. Bajoghli $\cdot$ N. Aghaallaei $\cdot$ T. Czerny $(\bowtie)$

Department for Biomedical Sciences,

University of Veterinary Medicine,

Veterinärplatz 1,

1210 Vienna, Austria

e-mail: thomas.czerny@vu-wien.ac.at

G. Jung $\cdot$ T. Czerny

Department for Applied Life Sciences, University of Applied Sciences,

FH Campus Wien, Viehmarktgasse 2A,

1030 Vienna, Austria
}

Present Address:

B. Bajoghli $\cdot$ N. Aghaallaei

Max-Planck Institute of Immunobiology,

Stuebeweg 51,

79108 Freiburg, Germany the otic-inducing properties of the hindbrain. However, signals different from Fgf3 and Fgf8 are necessary to explain the formation of the ectopic ear structures, suggesting that $\mathrm{Wnt}$ signalling is involved in the otic induction process in medaka.

Keywords Wnt1 · Otic induction · Cyclopic ear · Medaka

\section{Introduction}

In teleost fish, the inner ear originates from an ectodermal placode located at the level of rhombomere 4 (r4) of the hindbrain, and the otic placode then cavitates to form the otic vesicle (reviewed in Whitfield et al. 2002). Classical transplantation experiments demonstrated a prominent role of the hindbrain in the otic induction process. In zebrafish, grafting hindbrain tissue to the ventral side of the embryo induces ectopic otic vesicles (Woo and Fraser 1998). Members of the fibroblast growth factor (Fgf) family of secreted ligands are the best candidates for otic-inducing factors (reviewed in Riley and Phillips 2003; Whitfield et al. 2002), but the role of other modulating factors such as Wnt signalling in this process is not clear.

Experiments with lithium chloride treatment by Gutknecht and Fritzsch (1990) in Xenopus embryos led to multiple expanded otic vesicles. Since lithium is a potent inhibitor of GSK3; these experiments can be interpreted as a first indication of otic-inducing effects by canonical Wnt signalling. A model for induction of the chick inner ear was proposed in which $F g f 19$ cooperates with $W n t 8 c$ to induce ectopic otic placodes (Ladher et al. 2000). Neither Fgf19 nor Wnt $8 \mathrm{c}$ was able to induce the expression of all otic markers in explants of uncommitted ectoderm, whereas a combination of both genes strongly induced the full range of markers genes. Loss of Wnt8 function experiments in zebrafish suggest 
that otic placode induction occurs normally in the absence of Wnt8 expression (Phillips et al. 2004). In that study, morpholino knockdown of the Wnt8 gene and misexpression of the Wnt antagonist dickkopf 1 failed to block otic induction. Phillips and colleagues proposed that Wnt serves to regulate the temporal expression of $F g f 3$ and $F g f 8$ in the hindbrain, hence playing an indirect role in otic induction (Phillips et al. 2004). Furthermore, Wnt signalling was proposed to affect the preplacodal state in chicks (Bailey and Streit 2006), and in mice, it was suggested to be required after Fgf-dependent induction to maintain otic fate (Ohyama et al. 2006). Here, we address the question whether Wnt signalling is involved in otic induction in medaka fish. We demonstrate that ectopic activation of this pathway affects otic development at the time of induction by juxtaposing regions normally separated in the embryo. This is in good agreement with hindbrain tissue as a source for otic-inducing signals. However our experiments suggest that signals in addition to Fgf3 and Fgf8 induce the formation of ectopic ear structures in medaka.

\section{Materials and methods}

Fish strain and transgenic lines

Embryos of the medaka Cab inbred strain were used for all experiments. Stages were determined according to Iwamatsu (2004). All studies on heat-inducible Wnt1-transgenic embryos were carried out using three independent lines described previously (Bajoghli et al. 2007).

\section{Embryo injections}

Medaka embryos were microinjected into single blastomeres at the one- to two-cell stage. mRNA of mouse Wnt1 was injected at $200 \mathrm{ng} / \mu \mathrm{l}$. For transient experiments, a heatinducible Wnt1 construct (Bajoghli et al. 2007) was injected at $10 \mathrm{ng} / \mu \mathrm{l}$ together with $I-S c e I$ meganuclease.

\section{Isolation of medaka EphA4 and Hoxbla probes}

The primers used for the RT-PCR were as follows: for EphA4, 5- GAAAAGAACATCCCCATTCG-3 and 5-GAGTTGCG TTCCTCATATCCT-3; for Hoxbla, 5-ATGGAAAACATG AACTCCTTTG-3 and 5-GTGCGGGACCGTTAGGTA-3. The DNA fragments were cloned into the pGEM-Teasy vector (Promega).

Whole-mount in situ hybridisation

Whole-mount in situ hybridisation was performed as described previously using DIG- or FITC-labelled probes (Aghaallaei et al. 2005).

\section{Results}

To elucidate the function of Wnt signalling during medaka otic induction, we decided to use gain-of-function analysis. Wnt1, Wnt8 and Wnt3a proteins define a functional class of Wnt ligands (Torres et al. 1996), which preferentially stimulate the canonical Wnt pathway (reviewed by Logan and Nusse 2004). We selected Wnt1 for our misexpression experiments. Injection of Wnt1 mRNA into medaka embryos resulted in a posteriorised phenotype characterised by severe truncations of anterior structures (Fig. 1b). Similar phenotypes have been observed upon ectopic activation of canonical Wnt signalling in various vertebrate species (Kelly et al. 1995; Stachel et al. 1993; van de Water et al. 2001; Yamaguchi and Shinagawa 1989). Since Wnt signalling has multiple functions during early embryonic development depending on the time window (Liu et al. 2005), we switched to induced expression using a heat-shock-inducible system (Bajoghli et al. 2004). Activation of the injected heat-shock Wntl construct at early gastrulation resulted in the same posteriorisation phenotype seen for mRNA. However, $18 \%$ of the embryos (13 of 70) showed enlarged otic vesicles (data not shown), and in addition, $7 \%$ of the embryos (five of 70 ) exhibited small ectopic otic vesicles located anteriorly to the endogenous otic vesicle (Fig 1c, arrow). When we repeated these experiments with an expression construct for the zebrafish $W n t 8$ gene, we also observed ectopic otic vesicles in $20 \%$ of the embryos (11 of 56; data not shown). These experiments are consistent with data for injection of a Wnt8 expression construct in zebrafish (Phillips et al. 2004). In order to overcome the mosaic nature of the injection technique, we repeated the experiments with a heat-shock $W n t 1$ transgenic medaka line (Bajoghli et al. 2007). In agreement with transient misexpression, uniform activation of Wntl in the whole embryo at early gastrulation led to posteriorisation. However, instead of the isolated ectopic vesicles observed before, one large vesicle appeared in the Wnt 1 -transgenic embryos, covering the complete anterior region starting from the position of the endogenous otic vesicles (Fig 1d). At later stages, the vesicle forms inner ear structures and contains multiple otoliths (Supplementary Fig. S1). We confirmed the identity of this ectopic otic structure by marker gene expression. The expression patterns of Starmaker (Fig. 1f), Eyal and Sixl identified the structures as otic vesicles (Supplementary Fig. S1). Due its similarity with cyclopic eyes, we named this phenotype "cyclopic ear".

In order to learn more about the effect of timing, we performed a time-course experiment by systematically varying the time of heat-shock activation in Wntl transgenic embryos from $30 \%$ epiboly to the three-somite stage (Fig. 2a). The experiments indicate a strict time dependence on the extent of the anterior truncations. Early activation of Wnt signalling leads to a severe truncation of the body axis 
Fig. 1 Activation of the canonical Wnt pathway in medaka embryos. Dorsal views of embryos at $2 \operatorname{dpf}(\mathbf{a}, \mathbf{c}, \mathbf{d})$ and $5 \mathrm{dpf}$ (b). A non-transgenic sibling (a) is compared to embryos with transient injections of Wnt 1 mRNA (b), a heat-inducible Wntl construct (c) and Wnt1-transgenic lines (d). Starmaker is strongly expressed in normal otic vesicles (e) and in cyclopic ear structures (f). An ectopic otic vesicle is indicated by an arrow. The heat-shock treatment was performed for $2 \mathrm{~h}$ at $39^{\circ} \mathrm{C}$ for $\mathbf{c}$, $\mathbf{d}$ and $\mathbf{f}$ and $3 \mathrm{~h}$ at $30 \%$ for a. ey eye, $o v$ otic vesicle, $p f$ pectoral fin
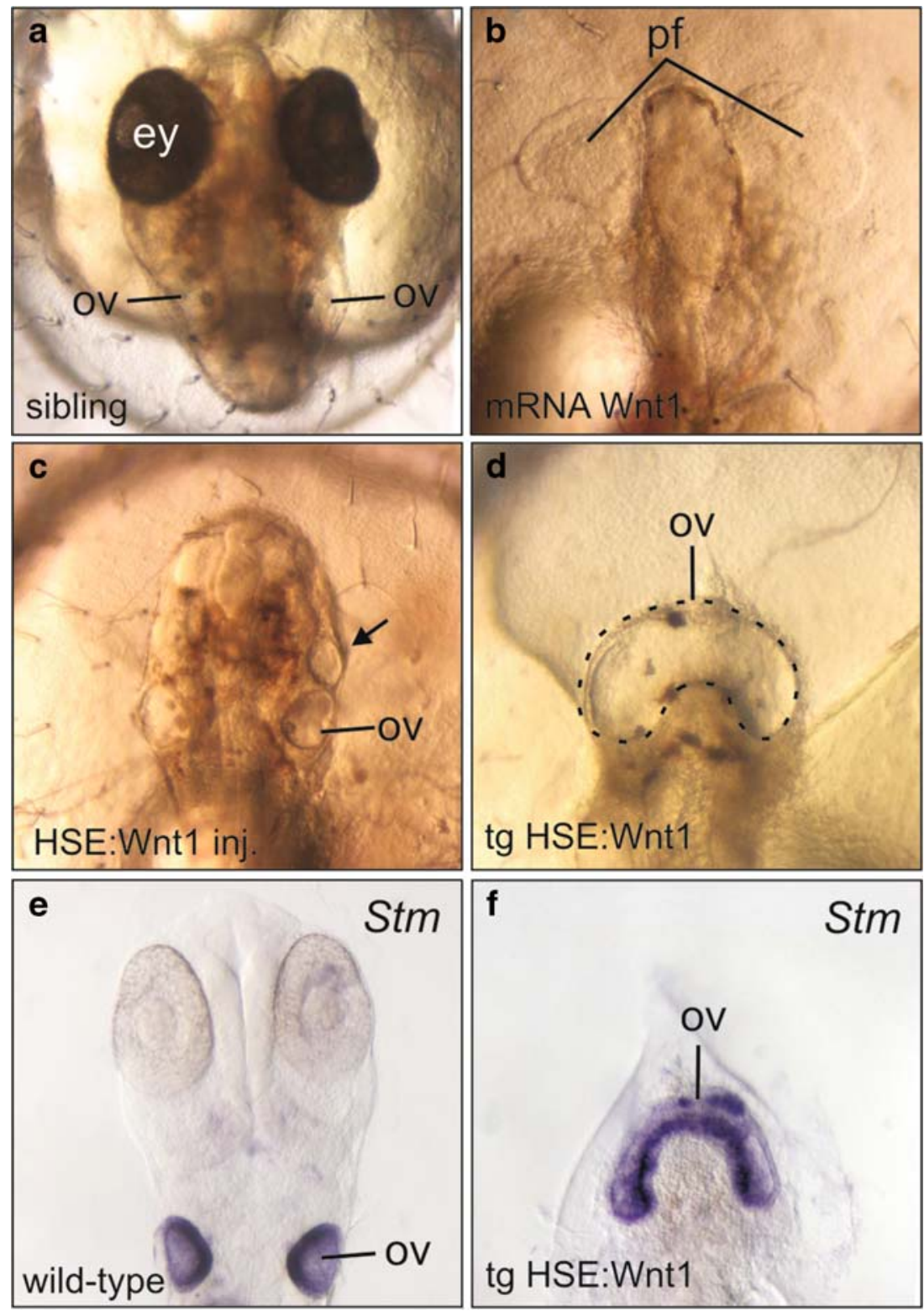

at the level of the hindbrain, whereas posterior parts of the embryos are not affected. The extent of the axis truncations does not differ dramatically between the one cell stage (mRNA injection, see Fig 1b) and early gastrulation (Fig. 2a). However between early and late gastrulation, the truncations are gradually reduced, with midbrain structures appearing at heat shocks at 60\% epiboly and forebrain structures at $80 \%$ epiboly. Ectopic Wnt activation at neurula stages only affects eye development, whereas embryos heat-shocked during somitogenesis appear normal. These phenotypes would correlate with the timing of neural plate induction by the migrating mesoderm, which might be blocked at different time points by the ectopic Wnt signals. The gradual loss of anterior structures can also be observed with hindbrain marker gene expression (see below).
In the time-course experiment, the cyclopic ear phenotype was only observed when Wnt signalling was induced at $30 \%$ epiboly. Slightly later activation of the pathway at $40 \%$ epiboly generates embryos with normal otic vesicle morphology. In previous work, we identified early gastrulation as a critical phase for otic induction in medaka (Aghaallaei et al. 2007). The ectopic Wnt activity therefore affects otic development during the induction phase.

To examine whether the effect of Wnt signalling is dosage dependent, we varied the time of heat treatment in embryos at early gastrulation from $5 \mathrm{~min}$ to $2 \mathrm{~h}$. We used the Gfp signals originating from the bidirectional heatshock promoter as a marker for the expression level (Fig. 2b). Interestingly, all embryos heat-treated for more than 10 min showed the same phenotype; the only 
A

Stage of heat tratment $\left(2 \mathrm{~h} / 39^{\circ} \mathrm{C}\right)$
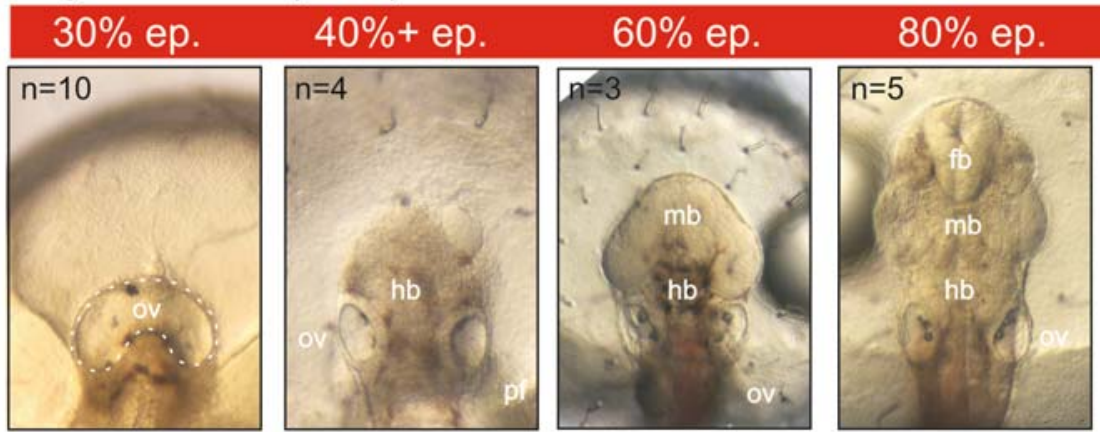

neurula
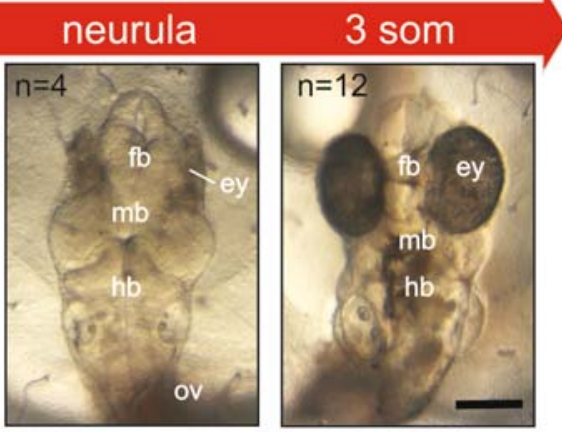

B
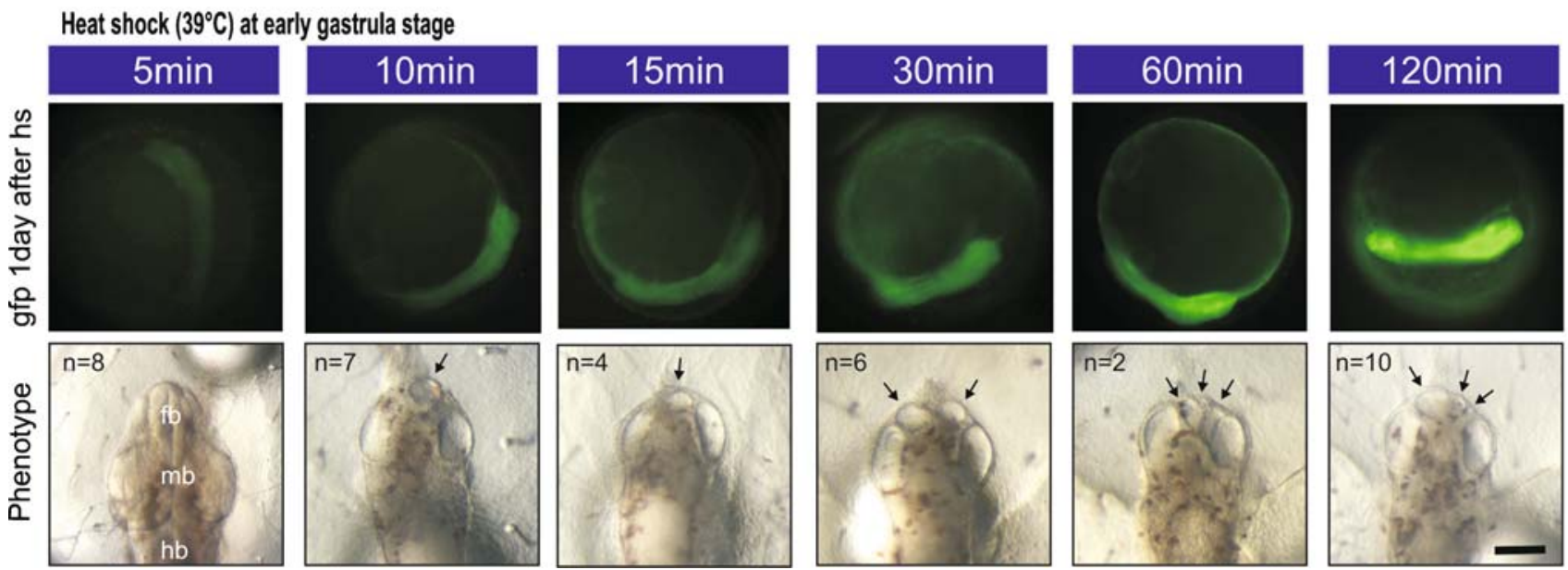

different durations. The Gfp activity 1 day after heat shock was used as a marker. Ectopic otic vesicles are indicated by arrows. $n$ the number of examined embryos, ep. epiboly, ey eye, $f b$ forebrain, $m d$ midbrain, $h b$ hindbrain, $o v$ otic vesicle

at $50 \%$ epiboly, which did not develop ectopic otic vesicles. The embryos were fixed at a preplacodal stage (two-somite stage). For analysis of the hindbrain structures, we used probes directed against EphA4 and Hoxbla, expressed in $\mathrm{r} 3 / \mathrm{r} 5$ and $\mathrm{r} 4$, respectively (see "Materials and methods"; Fig 4a). The expression patterns of the hindbrain marker genes confirmed the truncations of the anteroposterior axis in the Wnt1 transgenic embryos. In group B embryos, r3 and all structures posterior to this rhombomere appeared normal (Fig. 4c, f, i, r), whereas in group A embryos, the last distinguishable rhombomere was $\mathrm{r} 5$ (Fig. 4b, e, h). Interestingly, the expression patterns of the marker genes suggest that the remaining anterior structures of the embryos are of one uniform fate. In group A embryos, this region anterior to r5 expressed Hoxla, Fgf8 and Fgf3 (Fig. 4b, e, h), which would be consistent with an $\mathrm{r} 4$ fate. In group B embryos, the region anterior to $\mathrm{r} 3$ expressed $\mathrm{Fg} f 8$ (Fig. 4f), indicating an $\mathrm{r} 2$ fate. However, we also found expression of $\mathrm{Fgf3}$ and Engrailed 2 (En2; Fig. 4i, r), normally not expressed in r2. In wild-type embryos, these 

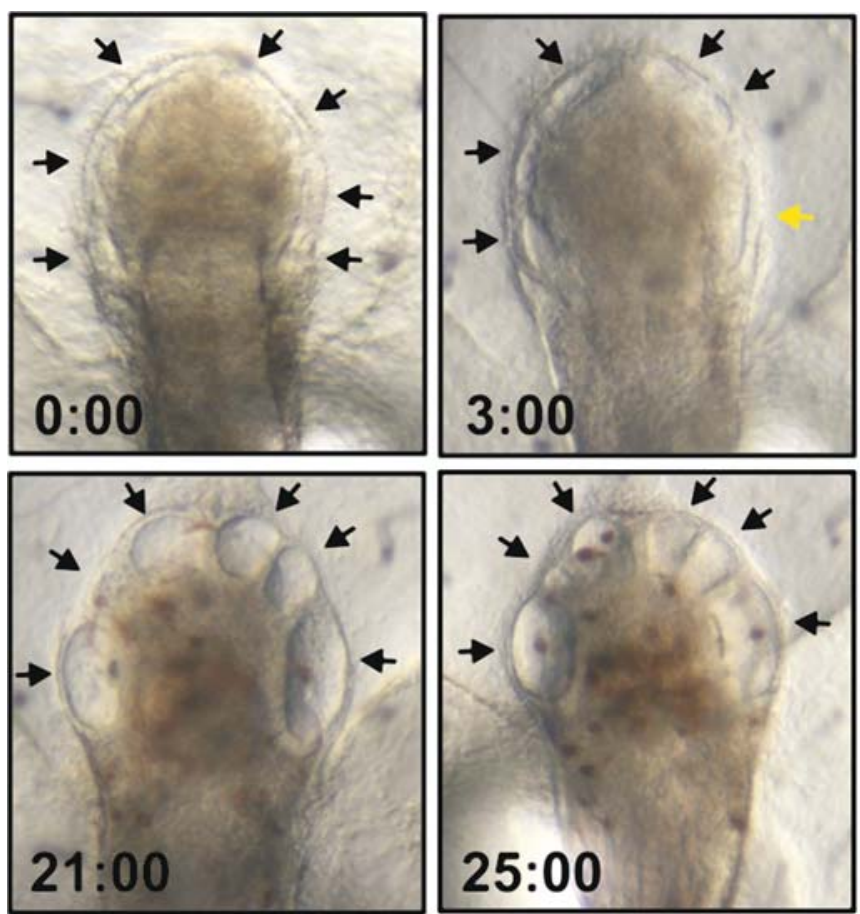

Fig. 3 Time-lapse analysis of the cyclopic ear phenotype. The otic vesicle morphology was analysed in Wnt 1 -transgenic embryos from stage 20 to stage 30 . Heat shock was performed at $30 \%$ epiboly for $2 \mathrm{~h}$

two genes are expressed in the more anteriorly positioned midbrain-hindbrain boundary (mhb). The fate of the region anterior to $\mathrm{r} 3$ in group $\mathrm{B}$ embryos therefore remains unclear.

A combination of local competence factors together with signals from a distance is thought to induce otic structures in vertebrates (Riley and Phillips 2003; Whitfield et al. 2002). Fgf ligands are thought to mediate the signals originating from $\mathrm{r} 4$ (Maves et al. 2002; Phillips et al. 2001), and Dlx3b and Foxil are the best candidates for the competence factors (Esterberg and Fritz 2009; Lee et al. 2003; Solomon et al. 2004). Dlx3b is a preplacodal marker gene (Liu et al. 2003) initially expressed in a horseshoeshape surrounding the entire neural plate (Supplementary Fig. S3; Hochmann et al. 2007; Kaji and Artinger 2004). Due to the anterior truncations, the neural plate was shortened in the Wnt 1 embryos, but Dlx $3 b$ expression in the adjacent ectoderm remained in both groups (Fig. 4k, 1 and Supplementary Fig. S3). In group A embryos, the expression was upregulated in the anterior region (Fig. 4k), anticipating the development of the ectopic otic structures. In group $\mathrm{B}$, strong $D l \times 3 b$ expression was restricted to regions flanking $\mathrm{r} 4$, coinciding with the normal position of otic structures (Fig. 41). The forkhead-domain containing transcription factor Foxil is expressed in two domains positioned laterally to the neural plate (Hochmann et al. 2007), which we also found in group B embryos (Fig. 4o).
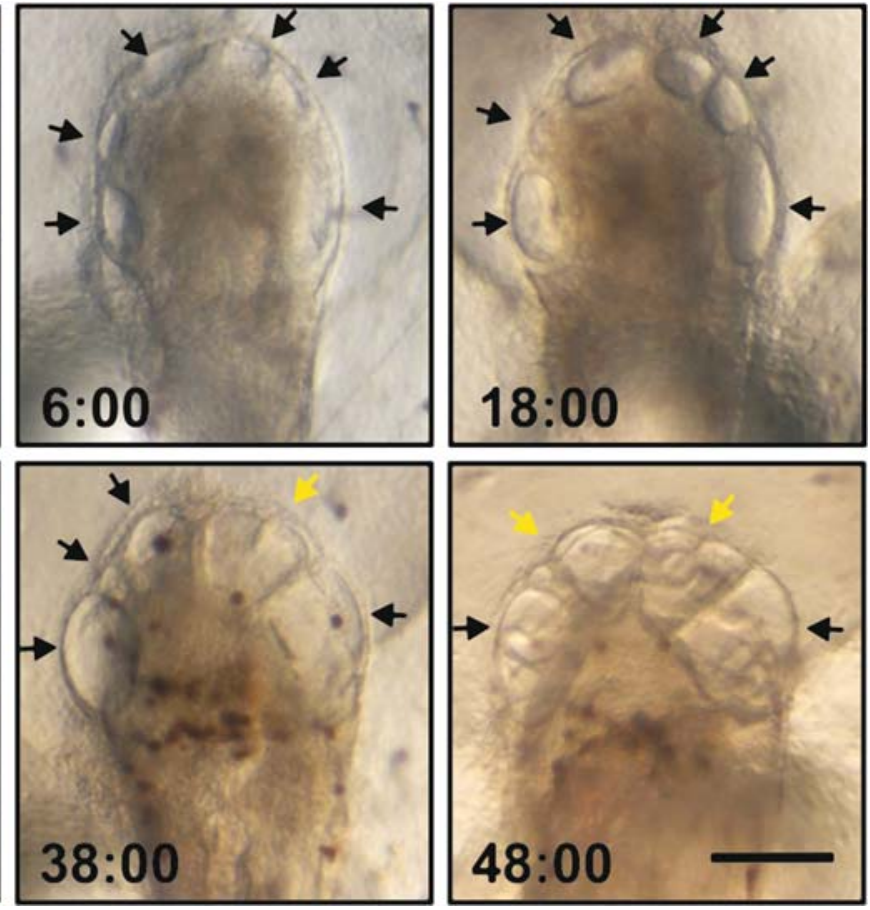

at $39^{\circ} \mathrm{C}$. Ectopic otic vesicles are indicated by black arrows. The fusion of vesicles is indicated by yellow arrows

Interestingly we detected strong Foxil expression in group A embryos at the position where the ectopic otic structures develop (Fig. 4n), contrary to group B embryos, which do not show any Foxil expression in this region (Fig. 4o).

Sox3 is a marker for both the epibranchial and the otic preplacodal region, whereas Pax2 marks otic tissue specifically (Nikaido et al. 2007). In order to see whether the ectopic placodes of Wnt 1 expressing embryos are restricted to otic development, we analysed group A embryos for expression of the two marker genes (Supplementary Fig. S4). We found both genes activated in overlapping anterior regions, indicating a clear bias towards ear development. This is in agreement with data from chick and mouse experiments, where Wnt signalling was found to partition the progenitor region, positively into inner ear and negatively into epibranchial placodes (Freter et al. 2008; Ohyama et al. 2006). Group B embryos did not show expression of either marker gene in the anterior region (data not shown).

\section{Discussion}

Ectopic expression of Wnt 1 during early development of vertebrates is known to result in anterior truncations (Christian and Moon 1993). The heat-shock transgenic line allowed us to perform highly specific time-course experiments for ectopic Wntl activity in medaka fish. By 


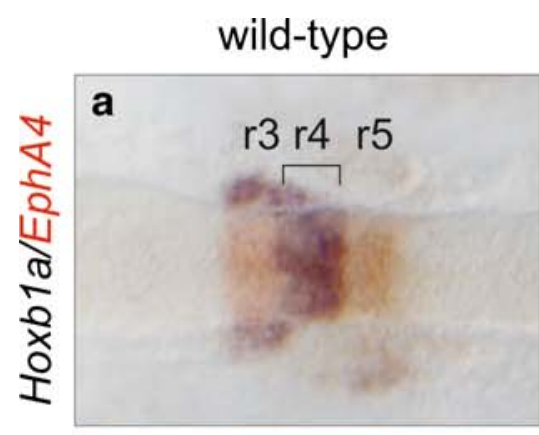

group A (HS 30\% ep) group B (HS 50\% ep)
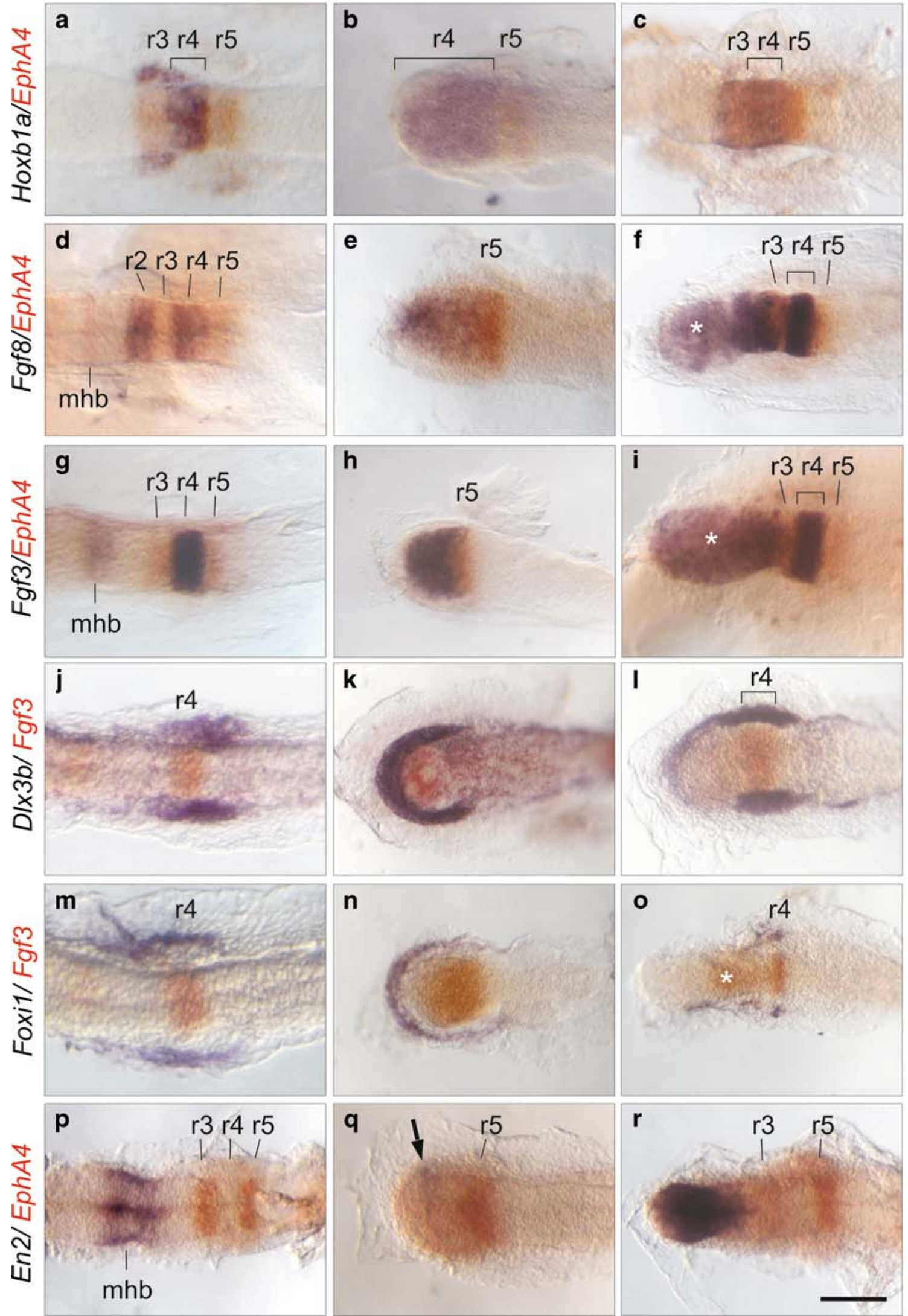
Fig. 4 Analysis of hindbrain and otic marker genes in the Wnt1transgenic lines. Dorsal views of embryos at two somites $(\mathbf{a}-\mathbf{r})$. Anterior is to the left. The positions of rhombomeres $(r)$ and the midhindbrain boundary $(\mathrm{mhb})$ are indicated. The heat shock of Wntltransgenic embryos was performed at $30 \%$ epiboly in group A and $50 \%$ epiboly in group B, respectively. The asterisks indicate anterior expression of $F g f 3$ and $F g f 8$, respectively, in group B embryos, despite the fact that they lack ectopic ear structures. The arrow indicates weak En2 expression. mhb. mid-hindbrain boundary

systematically varying the time of activation, we observed a strict dependence of timing on the extent of the anterior truncations (Fig. 2a). These phenotypes can be interpreted by a gradient of Wnt signalling being responsible for patterning the anteroposterior axis (Kiecker and Niehrs 2001). The critical influence of timing in our experiments is in good agreement with lithium exposure experiments (Yamaguchi and Shinagawa 1989).

Induction of canonical Wnt signalling in the transgenic embryos at $30 \%$ epiboly resulted in a novel phenotype. Extensive otic tissue developed and finally covered the complete anterior region of the embryos. Interestingly, these effects on otic induction seemed to be uncoupled from the axis truncations. Injection of both Wnt1 mRNA and DNA resulted in truncations, but only the uniform misexpression at $30 \%$ epiboly in the transgenic line produced the cyclopic ear phenotype. In addition, the number of ectopic otic vesicles was dose dependent, whereas the axis truncations appeared indistinguishable from low to high $W n t 1$ doses (Fig. 2b). In the zebrafish model system, activation of canonical Wnt signalling at the midblastula transition did not produce ectopic otic vesicles. This experiment was used as an argument against a role of canonical Wnt signalling in otic induction (Phillips et al. 2004). In support of these data, we also failed to induce ectopic otic tissue with constitutive active DNA constructs in medaka. However, in contrast to the latter study, we demonstrate a strict time dependence for Wnt signalling in the otic induction process, namely at $30 \%$ epiboly.

Fgf signalling plays a major role in otic induction (reviewed in Riley and Phillips 2003; Whitfield et al. 2002), and we recently could demonstrate an inducing effect of $F g f 8$ misexpression during early gastrulation in medaka. In these experiments, the formation of ectopic otic vesicles was further boosted by coexpression of the competence factors $D l x 3 b$ and Foxil (Aghaallaei et al. 2007). Here we compared groups of Wnt1-transgenic embryos induced at 30\% epiboly (group A), with those induced at $50 \%$ epiboly (group B). In both groups of Wnt1transgenic embryos, we found similar expression of Fgf8 (Fig. 4e, f); however, only group A embryos developed ectopic otic structures. Fgf3 expression was stronger in the anterior region of group A embryos (Fig. 4h, k, n) and weaker and more variable in group B (Fig. 4i, 1, o). In addition, we observed $D l x 3 b$ activity in both groups (Fig. 4k, 1 and Supplementary Fig. S3). Therefore Fgf signalling activity and $D l x 3 b$ expression, representing two prerequisites for otic induction, were present in the anterior regions of both groups. However, in group B embryos, no Foxil expression was detected in this region (Fig. 4o), contrary to group A (Fig. 4n). The differential Foxil expression in the two groups of embryos could be critical for the induction of ectopic otic development (Fig. 4n, o). Indeed we previously found a central role for this competence factor in the preplacodal gene regulatory network in medaka (Aghaallaei et al. 2007). Loss-offunction experiments in zebrafish also confirm a critical role of Foxil (Lee et al. 2003; Solomon et al. 2003).

The main result of pulsed Wnt activation is the truncation of anterior structures. In order to study the role of canonical Wnt signalling on inner ear formation, it would be ideal to uncouple the effects on the otic induction process from those on axis formation. However, this is not possible with our experimental approach. Due to the axis truncations, an expanded $\mathrm{r} 4$ region is positioned next to the anterior expression domains of Dlx $3 b$ and Foxil in group A embryos. The close contact between this rhombomere and ectoderm positive for the competence factors is a hallmark of normal otic development. The ectopic activation of Foxil in group A embryos could be mediated by signals of this $\mathrm{r} 4$ region. In agreement with this, we previously described the activation of Foxil by Fgf8 in medaka embryos (Aghaallaei et al. 2007). However, we found equal expression of $F g f 8$ in both group A and group B embryos (Fig. 4e, f). Anterior Fgf3 expression was variable in group B (Fig. 4i, 1, o), but since none of these embryos developed ectopic ear structures, $F g f 3$ expression is unlikely to be responsible for the differences. Therefore signals in addition to these Fgf ligands seem to be essential for the ectopic otic development in group A embryos. Alternatively, signals could be secreted from the hindbrain structures in group B embryos, which block the formation of otic tissue, but instead promote the midbrain-hindbrain boundary gene Engrailed 2. Interestingly, recent experiments with retinoic acid (RA) signalling in zebrafish revealed widespread ectopic otic induction upon excess activation of this pathway (Hans and Westerfield 2007). Foxil was ectopically activated in these embryos, whereas other marker genes for otic induction were not altered significantly. It is therefore possible that a genetic network of different signalling pathways (Fgf/RA/ Wnt) controls otic induction and in particular Foxi1. Further experiments will be necessary to clarify the role of Wnt signalling in this process.

Acknowledgements We thank Jochen Wittbrodt and Mirana Ramialison for the medaka Starmaker probe, which was kindly provided before publishing. The work was supported by the Austrian Science Fund (FWF, grant 19571-B11). 
Open Access This article is distributed under the terms of the Creative Commons Attribution Noncommercial License which permits any noncommercial use, distribution, and reproduction in any medium, provided the original author(s) and source are credited.

\section{References}

Aghaallaei N, Bajoghli B, Walter I, Czerny T (2005) Duplicated members of the Groucho/Tle gene family in fish. Dev Dyn 234:143-50

Aghaallaei N, Bajoghli B, Czerny T (2007) Distinct roles of Fgf8, Foxi1, Dlx3b and Pax8/2 during otic vesicle induction and maintenance in medaka. Dev Biol 307:408-20

Bailey AP, Streit A (2006) Sensory organs: making and breaking the pre-placodal region. Curr Top Dev Biol 72:167-204

Bajoghli B, Aghaallaei N, Heimbucher T, Czerny T (2004) An artificial promoter construct for heat-inducible misexpression during fish embryogenesis. Dev Biol 271:416-30

Bajoghli B, Aghaallaei N, Soroldoni D, Czerny T (2007) The roles of Groucho/Tle in left-right asymmetry and Kupffer's vesicle organogenesis. Dev Biol 303:347-61

Christian JL, Moon RT (1993) Interactions between Xwnt-8 and Spemann organizer signaling pathways generate dorsoventral pattern in the embryonic mesoderm of Xenopus. Genes Dev 7:13-28

Esterberg R, Fritz A (2009) dlx3b/4b are required for the formation of the preplacodal region and otic placode through local modulation of BMP activity. Dev Biol 325:189-99

Freter S, Muta Y, Mak SS, Rinkwitz S, Ladher RK (2008) Progressive restriction of otic fate: the role of FGF and Wnt in resolving inner ear potential. Development 135:3415-24

Gutknecht D, Fritzsch B (1990) Lithium can transform ear placodes of Xenopus into multiple otic vesicles connected by tubes. Naturwissenschaften 77:235-7

Hans S, Westerfield M (2007) Changes in retinoic acid signaling alter otic patterning. Development 134:2449-58

Hochmann S, Aghaallaei N, Bajoghli B, Soroldoni D, Carl M, Czerny $\mathrm{T}$ (2007) Expression of marker genes during early ear development in medaka. Gene Expr Patterns 7:355-62

Iwamatsu T (2004) Stages of normal development in the medaka Oryzias latipes. Mech Dev 121:605-18

Kaji T, Artinger KB (2004) dlx $3 b$ and dlx4b function in the development of Rohon-Beard sensory neurons and trigeminal placode in the zebrafish neurula. Dev Biol 276:523-40

Kelly GM, Erezyilmaz DF, Moon RT (1995) Induction of a secondary embryonic axis in zebrafish occurs following the overexpression of beta-catenin. Mech Dev 53:261-73

Kiecker C, Niehrs C (2001) A morphogen gradient of Wnt/betacatenin signalling regulates anteroposterior neural patterning in Xenopus. Development 128:4189-201

Ladher RK, Anakwe KU, Gurney AL, Schoenwolf GC, Francis-West PH (2000) Identification of synergistic signals initiating inner ear development. Science 290:1965-7
Lee SA, Shen EL, Fiser A, Sali A, Guo S (2003) The zebrafish forkhead transcription factor Foxil specifies epibranchial placode-derived sensory neurons. Development 130:2669-79

Liu D, Chu H, Maves L, Yan YL, Morcos PA, Postlethwait JH, Westerfield M (2003) Fgf3 and Fgf8 dependent and independent transcription factors are required for otic placode specification. Development 130:2213-24

Liu F, van den Broek O, Destree O, Hoppler S (2005) Distinct roles for Xenopus Tcf/Lef genes in mediating specific responses to Wnt/beta-catenin signalling in mesoderm development. Development 132:5375-85

Logan CY, Nusse R (2004) The Wnt signaling pathway in development and disease. Annu Rev Cell Dev Biol 20:781-810

Maves L, Jackman W, Kimmel CB (2002) FGF3 and FGF8 mediate a rhombomere 4 signaling activity in the zebrafish hindbrain. Development 129:3825-37

Nikaido M, Doi K, Shimizu T, Hibi M, Kikuchi Y, Yamasu K (2007) Initial specification of the epibranchial placode in zebrafish embryos depends on the fibroblast growth factor signal. Dev Dyn 236:564-71

Ohyama T, Mohamed OA, Taketo MM, Dufort D, Groves AK (2006) Wnt signals mediate a fate decision between otic placode and epidermis. Development 133:865-75

Phillips BT, Bolding K, Riley BB (2001) Zebrafish fgf3 and fgf8 encode redundant functions required for otic placode induction. Dev Biol 235:351-65

Phillips BT, Storch EM, Lekven AC, Riley BB (2004) A direct role for Fgf but not Wnt in otic placode induction. Development 131:923-31

Riley BB, Phillips BT (2003) Ringing in the new ear: resolution of cell interactions in otic development. Dev Biol 261:289-312

Solomon KS, Kudoh T, Dawid IB, Fritz A (2003) Zebrafish foxi1 mediates otic placode formation and jaw development. Development 130:929-40

Solomon KS, Kwak SJ, Fritz A (2004) Genetic interactions underlying otic placode induction and formation. Dev Dyn 230:419-33

Stachel SE, Grunwald DJ, Myers PZ (1993) Lithium perturbation and goosecoid expression identify a dorsal specification pathway in the pregastrula zebrafish. Development 117:1261-74

Torres M, Gomez-Pardo E, Gruss P (1996) Pax2 contributes to inner ear patterning and optic nerve trajectory. Development 122:3381-91

van de Water S, van de Wetering M, Joore J, Esseling J, Bink R, Clevers H, Zivkovic D (2001) Ectopic Wnt signal determines the eyeless phenotype of zebrafish masterblind mutant. Development 128:3877-88

Whitfield TT, Riley BB, Chiang MY, Phillips B (2002) Development of the zebrafish inner ear. Dev Dyn 223:427-58

Woo K, Fraser SE (1998) Specification of the hindbrain fate in the zebrafish. Dev Biol 197:283-96

Yamaguchi Y, Shinagawa A (1989) Marked alteration at midblastula transition in the effect of lithium on formation of the larval body pattern of Xenopus laevis. Dev Growth Differ 31:531-541 